\title{
THE DEVELOPMENT OF THE VIRTUAL DATA ACQUISITION AND ANALYSIS SYSTEM
}

\author{
Xiumin Yang, Weihua Tian, Ling Jiang, Yongshu Guo, Bo Wan \\ Dept. of Automatic Control, Shenyang Institute of Engineering, Shenyang, 110136,China; \\ Email: yxiumin@tom.com.
}

Abstract: A virtual multi-channel data acquisition system is developed based on computer technology and the virtual instrument development platform of LabWindows/CVI of NI Company of America. The PCI-6024E data acquisition card of NI Company of America is used as gateway device, and the Data Acquisition Library and data $\mathrm{I} / \mathrm{O}$ function library (I/O for DAQ) provided by Lab Windows/CVI of NI company are used to abbreviate programs to realize data acquisition of the real physical signals and do frequency analysis. This paper introduces the technology of hardware configuration, data acquisition, waveforms display and so forth of the virtual data acquisition system. And an analysis is done about the time domain analysis module. Through testing proof, this virtual instrument system can realize the function of traditional data collection system completely, has more strong function even.

Key words: Data Acquisition, Waveforms Display, Virtual Instrument, Spectrum Analysis.

\section{INTRODUCTION}

The hardware platform of the virtual instrument is composed of two parts, the computer and the interface equipment of $\mathrm{I} / \mathrm{O}$. The interface equipment of $\mathrm{I} / \mathrm{O}$, mainly realize data acquisition, magnifying and $\mathrm{A} / \mathrm{D}$ transformation of input signals. For a single-device virtual instrument, The interface equipment of $\mathrm{I} / \mathrm{O}$ of hardware disposition is a data acquisition card. And for an measuring system composed of multiple virtual instruments, the interface equipment of $\mathrm{I} / \mathrm{O}$ of hardware platform are buses including GPIB/GPIB488.2, RS232, VISA and VXI, etc.

This paper mainly proposes a design of single-device virtual data acquisition system, and a data acquisition card is adopted as interface

This project is supported by the Academic Science Research Fund of Liaoning Education Office of China No. 202263360.

Please use the following format when citing this chapter:

Yang, Xiumin, Tian, Weihua, Jiang, Ling, Guo, Yongshu, Wan, Bo, 2006, in International Federation for Information Processing (IFIP), Volume 207, Knowledge Enterprise: Intelligent Strategies In Product Design, Manufacturing, and Management, eds. K. Wang, Kovacs G., Wozny M., Fang M., (Boston: Springer), pp. 269-274. 
equipment of I/O. The card is composed of 4 parts: multichannel switch, amplifier, sampling/holding element and A/D transformer. Besides these, some other circuits as well as timing counter and bus interface circuit are also involved to complete data acquisition, amplification and $\mathrm{A} / \mathrm{D}$ transformation ${ }^{[1]}$.

Based on the proper choice of data acquisition card, a virtual data acquisition and analysis system is developed by using the Data Acquisition Library and $\mathrm{I} / \mathrm{O}$ function library (I/O for $\mathrm{DAQ}$ ) of virtual instrument developing platform of LabWindows/CVI of NI Company of America. The system can realize acquisition of real physical signals, and display, analysis, storage and printing of the data curves.

\section{DEVELOPMENT PLATFORM OF DATA ACQUISITION SYSTEM}

\subsection{Choice of data acquisition card}

The proposed data acquisition system is mainly designed to realize analog signals acquisition of the output of secondary appearance. Considering the requirements of usual data acquisition system to sampling frequency, speed, resolution capacity and number of channels as well as comparison of performance and cost, we finally choose PCI-6024E data acquisition card of NI company. PCI-6024E has $68 \mathrm{l} / \mathrm{O}$ connecting foots and can provide high performance and reliable function of data acquisition. The specific performance indexes and parameters are as follows ${ }^{[2,3]}$.

Analog inputs: 16 single inputs or 8 differential inputs

Input precision (bit): 12

Input range( $(\mathrm{V}): \pm 10, \pm 5$

Input gain: $0.5,1,10,100$

Sampling rate (S/s): $200 \mathrm{~K}$

Analog output: 2

Analog output precision (bit): 12

Digital I/O: 8

Counting timer: 2

\subsection{Software development of the data acquisition system}

There is two kinds of sampling technologies can be adopted: real time sampling and equivalent time sampling. Real time sampling is that the acquisitions of all sampling points of a signal are done during one acquisition process, and the sampled signal may be instant or periodic. Real 
time sampling is the most usual sampling mode of a data acquisition system. Under this mode, A/D transformer can once complete sampling of the whole input wave. This property is very fit for acquisition of single signal.

When doing data acquisition, DAQ (Data Acquisition) should be set first. LabWindows/CVI provides driving program libraries for various outside equipment ${ }^{[3]}$, by which the distribution and initiation of each kind of device can be finished conveniently. When setting DAQ, naming numbers of devices and input channels, choosing types of input signals and limiting data values need to be done. Then, Data Acquisition Library functions should be called to realize triggering control, channels choosing control and time bases control.

Waveform display module is mainly to display the waveforms of sampled data. This data acquisition system can accomplish acquisition and display of 8 paths of signals and do frequency analysis at one time. Two channels $A$ and $B$ should be chosen at random, and the waveforms of the results of $\mathrm{A}+\mathrm{B}$ or $\mathrm{A}-\mathrm{B}$ as well as the corresponding frequency analysis can be displayed.

According to the difference of display modes, the programs are distinguished in design. In the part of waveform display, display modes can be divided into 2 sorts according to the realizing principle. The first sort is double channel, under which the 8 channels can be displayed at one time. The second sort is single channel, under which only one of $A+B$ and A-B can be displayed. Because the graph controls can' $t$ support two formats of data, for the two different types of graphs, each uses a graph control to display. Some treatment is needed in users preface. The "Visible" property is adopted to control whether it is visible and ensure that every time there is only one graph displayed and the other is invisible.

Command controls include acquisition control button, pause button and waveform display button, etc. These controls are all located in Controls/Command. The acquisition control button controls data acquisition of the system. When it is pressed the acquisition module is called. And when the pause button is in use, the acquisition module is stopped working. After channel $A$ and $B$ are chosen and the button of $A+B$ or $A-B$ is pressed, then the waveform of $\mathrm{A}+\mathrm{B}$ or $\mathrm{A}-\mathrm{B}$ can be displayed. Besides, according to the catching function of the mouse, each acquisition point can be caught and its concrete value can be displayed by display control. It is two channels A and $B$ addition waveform as well as its waveform of frequency spectrum to show as Fig.1.

The panel of the system is showed in Fig.2. Channel 0 is a sine wave as input of the data acquisition system produced by standard signal generator; channel 1 is a square wave as input of the signal produced by the standard signal generator. The others are waveforms built by the return values of the 
arrays to produce waveforms of signal generator.
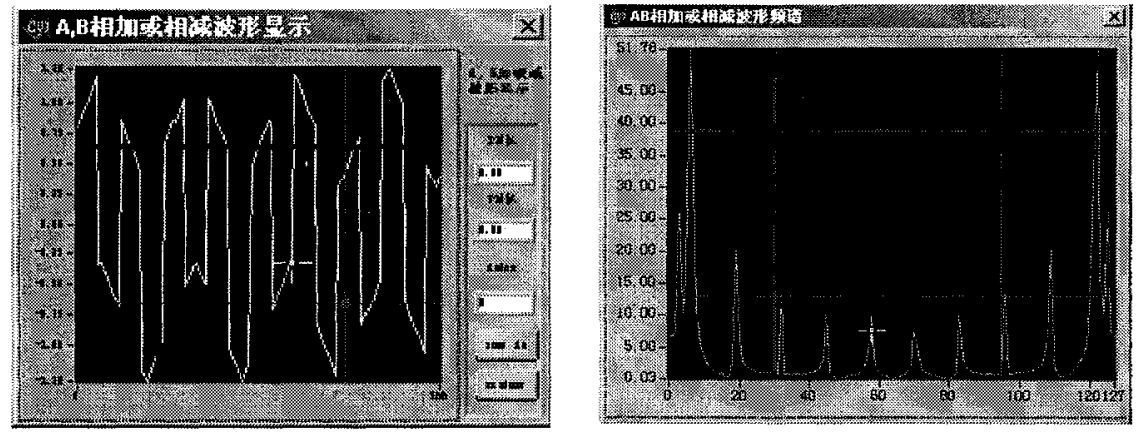

Figure 1. The superposition of square and sine waves and the frequency spectrum

The control parameters should be adjusted at suitable setpoints before the seed cleaner starts.

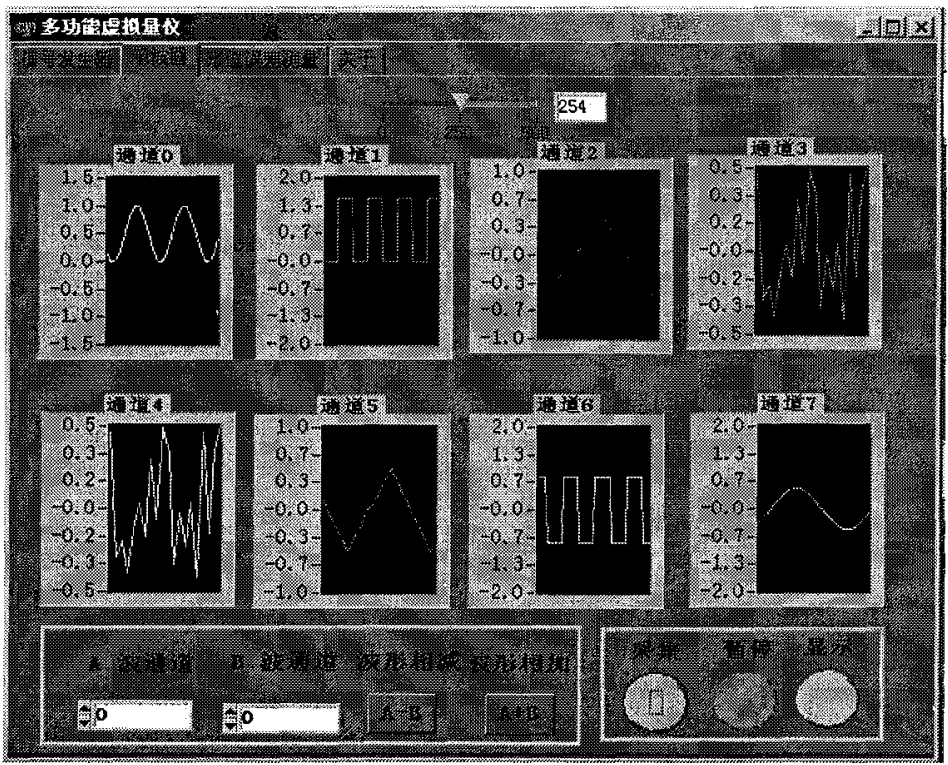

Figure 2. The panel of waveform displays

\section{SPECTRAL ANALYSIS MODULE}

There are two kinds of methods to observe signals. One is using oscillograph to do analysis in time domain. Here the oscillograph works at $\mathrm{V}-\mathrm{t}$ mode, displaying the waveform of the measured signal itself. It is 
necessary to denote the relationship of time and phase of the signal in concept of time domain, but it can' $t$ show the whole characters of the circuit. The second method is using frequency analysis instrument, which can separate each frequency level when testing power levels. Here the amplitude is displayed in frequency domain, and each frequency component of the signal can be directly observed. The power and voltage as functions of frequency can be displayed in curves.

If the sampling value of the measured signal $f(t)$ is known as $f_{k}$, then the frequency spectrum of $f(t)$ can be worked out by the computer through Fast Fourier Transformation. Here, low-pass filters, sampling circuits, A/D transformers and memories build up the data collecting system, which can transform the measured signals into digitals. With these data, the frequency spectrum of the measured signal is worked out by FFT calculator through Fast Fourier Transformation, and is showed on display.

Different kinds of standard values in frequency domain and the frequency component information can be acquired from doing frequency-domain analysis to signals. The most important analysis methods are Fast Fourier Transformation and its inverse transformation. The frequency-domain analysis methods in LabWindows/CVI mainly involve Fast Fourier Transformation function FFT( ), real number serial FFT function ReFFT( ), the corresponding inverse transformation functions InvFFT( ) and InvReFFT( ), totally 8 ones. Here, FFT( ) is adopted to realize the performance of frequency-domain analysis. It employs the split-base algorithm, which is the most effective currently and needs the least computing amount.

The result got from FFT( ) is a Plural number. Let $x$ and $y$ denote the real part and imaginary part of the spectrum, then the expressions of amplitude spectrum and phase spectrum are showed as (1) and (2).

$$
\begin{aligned}
& a m p=\sqrt{x^{2}+y^{2}} \\
& \text { phase }=\arctan \left(\frac{y}{x}\right)
\end{aligned}
$$

Seen from the expressions, the amplitude spectrum and phase spectrum are conformed after the Fourier frequency spectrum is worked out through FFT transformation.

The structure of a virtual frequency analysis system is similar to that of a virtual oscillograph system, but the data outlet of a frequency analysis system is mainly for spectrum analysis. The functions of a frequency analysis instrument must involve the data acquisition card to complete A/D sampling data input, the micro computer to do the consequent treatment and 
the proper display to realize functions of an oscillograph. Insertion or extraction to the input data at a certain multiple is needed to realize changing of the scan speed of consequent stage waveform. If it is required to change display amplitude, it is only needed to multiply the input data by corresponding coefficients.

To realize the functions of a frequency analysis instrument, the input data should be transformed from time domain to frequency domain first. The transforming method may be calling the corresponding digital signals processing modules such as the power-spectrum building module, and then the frequency analysis of the signal is done through programming. When calling the existing modules in Lab Windows/CVI, pay attention to the settings of some parameters.

The transformed data are already values in frequency domain. Take frequency as the axis of abscissas and make the data displayed, then the frequency spectrum graph is got. If it is together with local data insertion, extraction or being multiplied by corresponding coefficients, part of the spectrum graph can be magnified and deflated, and the information concerned in a certain frequency range can be analyzed in detail.

\section{CONCLUSION}

The proposed virtual data acquisition and analysis system can replace traditional measuring instruments and signal analysis and processing equipments. This system sufficiently embodies the flexibility of virtual instruments, and provides an instrument that has stronger functions and is simpler to operate. It is proved that the virtual system can completely realize the functions of a traditional data acquisition system, and has more strong function even.

Thanks for Jin Yinglian, graduate student of Northeast University, for her jobs in the development and debugging of the project.

\section{REFERENCES}

1. Yang Xiumin, Zhang Lei, Zhang Zhen, (2002), Calibration and analysis of the error for the forward channel of the measurement and control system, Microprocessors, vol. 93, pp. $53-55$.

2. National Instruments Corporation, (1998), Labwindows/CVI User Manual.

3. National Instruments Corporation, (1998), Labwindows/CVI Standard Libraries Reference Manual.

4. Yang Xiumin, Qin Hon, Zhang Xiguo, (2001), The design of the soft-panel for virtual instrument, Microprocessors, vol. 92, pp. 24-26.

5. Zhang Lei, Zhang Yu, Sun Baoshou, (2004), Development of the virtual roundness error measuring instrument, Machinery \& Electronics, vol. 134, pp. 60-62. 